\title{
Effectiveness of real-time tele-ultrasound for echocardiography in resource-limited medical teams
}

\author{
Tomohiro Kaneko ${ }^{1} \cdot$ Nobuyuki Kagiyama $^{2,3}$ (1) $\cdot$ Yutaka Nakamura ${ }^{2} \cdot$ Tomomi Hirasawa $^{4} \cdot$ Azusa Murata $^{2}$. \\ Ryoko Morimoto $^{2} \cdot$ Sakiko Miyazaki $^{1} \cdot$ Tohru Minamino $^{1}$
}

Received: 1 May 2021 / Revised: 25 June 2021 / Accepted: 29 July 2021 / Published online: 4 August 2021

(c) Japanese Society of Echocardiography 2021

\begin{abstract}
Background Echocardiography is a first-line tool for the screening of patients with cardiac dysfunction. However, the mastery of echocardiography requires significant training, and not all medical teams involve an echocardiography specialist. Telemedicine approaches can potentially improve the quality of echocardiography in resource-limited situations.

Methods We used a novel tablet-based hand-held ultrasound device that enables tele-ultrasound - a real-time video telecommunication with remote control for ultrasound images. A trainee scanned patients with various cardiovascular diseases and interpreted the images. Subsequently, the same trainee re-scanned the same patients and re-interpreted the same images using tele-ultrasound with an echocardiography specialist. An examination on the same patients by a blinded specialist was considered the gold standard.

Results We included 31 patients (median 77 [interquartile range 68-84] years old, $42 \%$ women). Mean absolute errors in left ventricular (LV) end-diastolic and end-systolic diameters, visual LV ejection fraction, and tricuspid annular plane systolic excursion decreased significantly after tele-ultrasound advice $(5.9 \mathrm{~mm}, 5.8 \mathrm{~mm}, 8.6 \%$, and 4.5 to $1.6 \mathrm{~mm}, 2.8 \mathrm{~mm}, 0.7 \%$, and $1.8 \mathrm{~mm}$, respectively, all $p<0.001)$, and intra-class correlation coefficients improved $(0.76,0.84,0.68$, and 0.44 to $0.96,0.93$, 0.99 , and 0.90 , respectively). Notably, with tele-advice, the trainee's examination showed perfect agreement with that of the specialist in classifying LV ejection fraction ( $>50 \%, 50-35 \%$, or $>35 \%$ ) and identifying significant valvular heart diseases. Conclusion Real-time tele-ultrasound improved a trainee's echocardiography results to those of a specialist-level examination. This approach might be helpful in resource-limited medical teams where echocardiographic specialists are not readily available.
\end{abstract}

Keywords Echocardiography $\cdot$ Telemedicine $\cdot$ Tele-ultrasound $\cdot$ Information and communication technology

\section{Abbreviations \\ ICT Information and communication technologies \\ ICC Intra-class correlation coefficient \\ LVIDd Left ventricular end-diastolic diameter}

Nobuyuki Kagiyama

kgnb_27_hot@yahoo.co.jp

1 Department of Cardiovascular Biology and Medicine, Juntendo University Graduate School of Medicine, Bunkyo-ku, Tokyo, Japan

2 Department of Cardiovascular Biology and Medicine, Juntendo University Faculty of Medicine, Bunkyo-ku, Tokyo, Japan

3 Department of Digital Health and Telemedicine R\&D, Juntendo University, 2-1-1 Hongo, Bunkyo-ku, Tokyo, Japan

4 Department of Nephrology, Juntendo University Faculty of Medicine, Bunkyo-ku, Tokyo, Japan
LVIDs Left ventricular end-systolic diameter

LAD Left atrial diameter

TAPSE Tricuspid annular plane systolic excursion

LVEF Left ventricular ejection fraction

LV Left ventricle

\section{Introduction}

Cardiovascular disease is one of the most prevalent and severe comorbidities in patients who require hospital admission, and there is a significant demand for evaluating cardiac function and status in various situations in hospitals and medical teams [1, 2]. Echocardiography is a first-line cardiac examination performed when cardiovascular complications are suspected, for example, in patients with abnormal electrocardiograms, elevated cardiac 
biomarkers, or diseases that frequently cause cardiovascular complications. However, significant training is required to perform and interpret echocardiography [3]; moreover, there are situations wherein an echocardiography specialist is not available, such as in small clinics, hospitals in rural areas, and infectious disease medical teams that restrict the number of participants.

Telemedicine refers to the remote delivery of healthcare services using information and communication technologies (ICT) [4]. Although the most common form of telemedicine is online clinics where patients directly talk with doctors online, such ICT approaches are also useful in communications and consultations between doctors [5, 6]. Recently, a novel ultrasound device that enables real-time screen sharing and telecommunication with remote doctors has emerged [7, 8]. This device provides a unique opportunity to perform "tele-ultrasound," a subfield of telemedicine, as an augmentation of non-specialist ultrasound by a real-time consultation with a specialist $[9,10]$. Such tele-ultrasound may be useful in resource-limited medical situations where a specialist is not readily available onsite, such as an infection disease team and a medical team that works in an ambulance/a medical helicopter where the number of medical staffs is restricted. In this study, we sought to elucidate the efficacy of real-time tele-ultrasound for echocardiographic examination in resource-limited medical teams.

\section{Methods}

\section{Study outline}

In November 2020, we conducted a study on consecutive patients with cardiovascular diseases who were admitted to Juntendo University Hospital. We simulated a resourcelimited medical team wherein only non-specialists could perform echocardiography, whereas a specialist was available online. Using a tablet-based handheld ultrasound device (Lumify; Philips, Andover MA), a trainee performed echocardiography on patients with various cardiovascular diseases and interpreted the echocardiographic images. Subsequently, the same trainee re-scanned and re-interpreted the same patients under tele-advice by a remote echocardiography specialist who was observing the echocardiographic images in another building. The device enables real-time telecommunication using an internet connection, and allows a remote doctor at distance to control a pointer on the ultrasound images that can be also visible to the examiner (Reacts system; INNOVATIVE IMAGING TECHNOLOGIES INC, Canada). The accuracies of the first (trainee alone) and the second examinations (under tele-advice) were assessed using the agreements with an examination by a blinded specialist, which was considered the gold standard.

\section{Level of training and echocardiography}

The trainees who participated in the study were in the middle of a general cardiology fellowship that was equivalent to level 1 of the training statement by American societies [3], which represented the basic training of competent consultants. They had just finished their 2-year residency program and had not undergone training on echocardiography. A specialist was defined as an attending echocardiography doctor who had finished training equivalent to level 3 training of the training statements by American societies. Using the hand-held device, left ventricular end-diastolic (LVIDd) and end-systolic diameter (LVIDs), left atrial diameter (LAD), and tricuspid annular plane systolic excursion (TAPSE) were measured. The handheld device was designed for point-ofcare ultrasound without a function for calculating left ventricular ejection fraction (LVEF) using the method of disks, and the purpose of the examination was not to obtain dedicated echocardiography-lab-level measurements; therefore, LVEF was assessed by visual estimation, which has been reported to be a good estimate of the method of disks if performed by experienced reviewers $[11,12]$. The severity of valvular heart disease was also investigated using color Doppler and B-mode images. Moderate and severe valvular heart diseases were considered significant valvular heart disease. All analyses were performed in accordance with the latest guidelines published by the American Society of Echocardiography and the Japanese Circulation Society [13-15].

\section{Statistical analysis}

The data are presented as the median [interquartile range] and frequency (\%) for continuous and categorical variables, respectively. Group differences were evaluated using the Mann-Whitney $U$ and chi-square or Fisher's exact tests for continuous and categorical variables, respectively. Errors of measurements were calculated as absolute values of the difference between trainee and specialist results, and paired $t$ tests were used to compare errors with and without tele-advice. Agreements between measurements were assessed using intra-class correlation coefficients (ICC; two-way random-effects, absolute agreements, for single rater) for continuous variables and weighted Kappa coefficients for categorical variables. Bland-Altman plots were used to check the limits of agreement.

All statistical analyses were performed using $\mathrm{R}$ version 4.0.3 (The R Foundation for Statistical Computing, Vienna, Austria), with packages "irr" and 
Fig. 1 Correlations between Trainees and Specialists. $x$ - and $y$-axes indicate the measurements obtained by a specialist and a trainee, respectively. The left and right panels show the correlations in the absence and presence of tele-ultrasound, respectively. Intra-class correlation coefficients improved for all parameters using tele-ultrasound. $L V$ left ventricular, ICC intra-class correlation coefficient

"BlandAltmanLeh" $[16,17]$. A two-tailed $p$ value $<0.05$ indicated statistical significance.

\section{Results}

\section{Population}

A total of 31 consecutive patients were scanned three times: by a trainee without tele-ultrasound, the same trainee

Table 1 Patient characteristics

\begin{tabular}{|c|c|}
\hline & $N=31$ \\
\hline Age & $77[68-84]$ \\
\hline Female & $13(42 \%)$ \\
\hline \multicolumn{2}{|l|}{ Etiology } \\
\hline Acute coronary syndrome & $2(6.5 \%)$ \\
\hline Arrhythmia & $3(9.7 \%)$ \\
\hline Heart failure & $24(77 \%)$ \\
\hline Peripheral artery disease & $2(6.5 \%)$ \\
\hline \multicolumn{2}{|l|}{ Rhythm } \\
\hline Sinus & $22(71 \%)$ \\
\hline Atrial fibrillation & $3(9.7 \%)$ \\
\hline Pacing & $6(19 \%)$ \\
\hline \multicolumn{2}{|l|}{ Device } \\
\hline PM/ICD & $6(19 \%)$ \\
\hline CRT-D/P & $3(9.7 \%)$ \\
\hline No device & $22(71 \%)$ \\
\hline Coronary artery disease & $10(32 \%)$ \\
\hline Prosthesis & $11(35 \%)$ \\
\hline \multicolumn{2}{|l|}{ Echocardiography } \\
\hline LV end-diastolic diameter, $\mathrm{mm}$ & $51[48-56]$ \\
\hline LV end-systolic diameter, mm & 33 [29-42] \\
\hline LV ejection fraction, $\%$ & $60[45-62]$ \\
\hline LA diameter, $\mathrm{mm}$ & $38[36-45]$ \\
\hline TAPSE, mm & $20.0[15.5-23.0]$ \\
\hline Valvular heart disease & $15(48 \%)$ \\
\hline Aortic stenosis & $2(6.5 \%)$ \\
\hline Aortic regurgitation & $6(19 \%)$ \\
\hline Mitral regurgitation & $11(35 \%)$ \\
\hline Tricuspid regurgitation & $7(23 \%)$ \\
\hline
\end{tabular}

$P M$ pacemaker, ICD implantable cardiac device, CRT-D cardiac resynchronization therapy defibrillator, $C R T-P$ cardiac resynchronization therapy pacemaker, $L V$ left ventricular, $L A$ left atrial, TAPSE tricuspid annular plane excursion
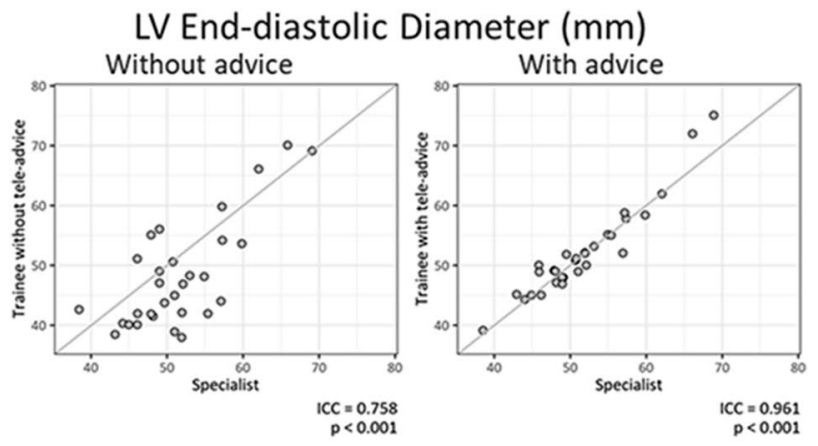

LV End-systolic Diameter (mm)

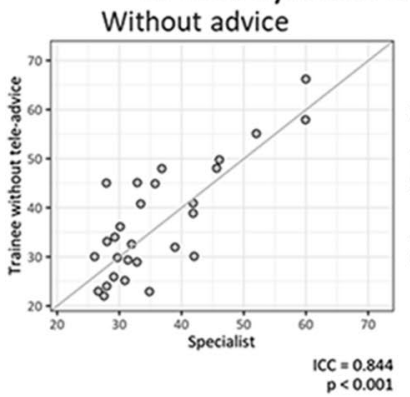

With advice

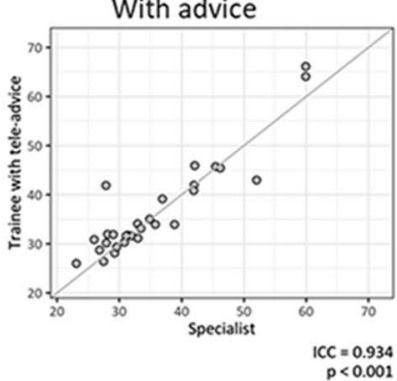

LV Ejection Fraction (\%)*
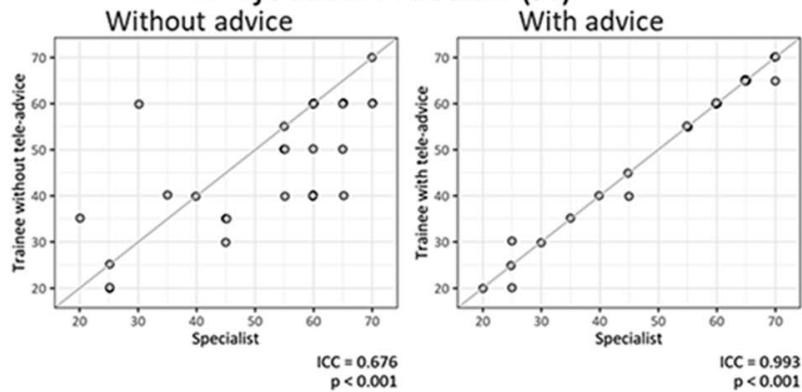

Left Atrial Diameter ( $\mathrm{mm})$
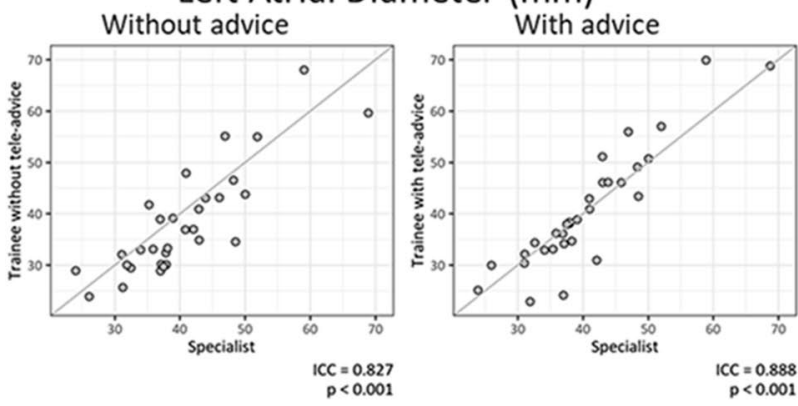

Tricuspid Annular Plane Excursion (mm)

Without advice
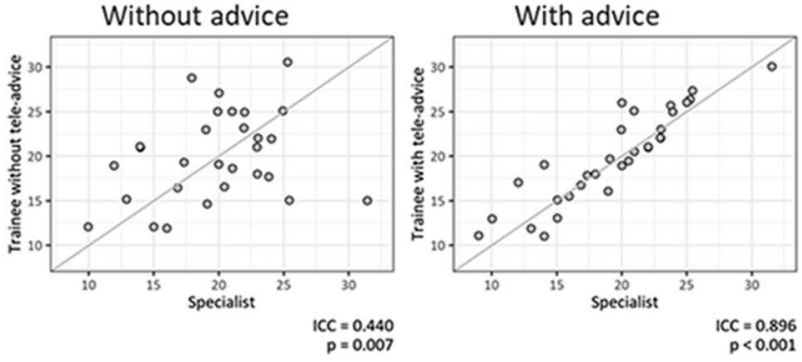
Fig. 2 Agreements between Trainees and Specialists. $x$-axes indicate the mean values of measurements obtained by a specialist and a trainee, whereas $y$-axes indicate the difference in the measurements (specialist minus trainee values). The left and right panels show the agreements in the absence and presence of tele-ultrasound, respectively. Limits of agreements shown in dashed lines are narrowed for all parameters by tele-ultrasound. $L V$ left ventricular

with tele-ultrasound, and a specialist. The median age was 77[68-84] years and $42 \%$ were women. Heart failure was the most frequent reason $(77 \%)$ for index hospitalization. The majority of patients had sinus rhythm, whereas $9.7 \%$ and $19 \%$ were atrial fibrillation and pacemaker rhythms, respectively. Approximately one-third of patients had an implanted cardiac device such as a pacemaker. Baseline and echocardiographic parameters obtained by a specialist are summarized in Table 1. Median values of left ventricular (LV) and left atrial diameters, LVEF, and TAPSE were normal; however, the interquartile ranges were beyond the normal range, suggesting that the population was heterogeneous. Approximately half of the patients had significant valvular heart diseases, and mitral regurgitation was most frequent in $35 \%$ of patients. There was only one patient with a small amount of pericardial effusion.

\section{Improvement of measurements by tele-ultrasound}

Without using the tele-ultrasound system, the trainee's scan showed moderate to good agreements with the specialist's measurements (Fig. 1, left side of each panel): ICC 0.76 for LVIDd, 0.84 for LVIDs, 0.68 for LVEF, 0.83 for LAD, and 0.44 for TAPSE. However, the limits of agreement (Fig. 2, left side of each panel) were wide, and the mean absolute errors were out of clinically acceptable ranges: LVIDd, 5.9 [ -9.3 to 15.4$] \mathrm{mm}$; LVIDs, 5.8 [ -14.9 to 13.4$] \mathrm{mm}$; LVEF, 8.6 [ -15.7 to 26.3 ]\%; LAD, 4.9 [ -8.5 to 13.0$] \mathrm{mm}$; and TAPSE, 4.5 [ -11.2 to 11.6$] \mathrm{mm}$. Under tele-advice, these agreements significantly improved (ICC 0.96 for LVIDd, 0.93 for LVIDs, 0.99 for LVEF, 0.89 for LAD, and 0.90 for TAPSE; Fig. 1, right side of each panel) and the limits of agreement decreased (Fig. 2, right side of each panel). The mean absolute errors significantly decreased under tele-advice, and most errors appeared clinically acceptable (1.6 mm for LVIDd, $2.8 \mathrm{~mm}$ for LVIDs, $0.7 \%$ for LVEF, $3.3 \mathrm{~mm}$ for LAD, and $1.8 \mathrm{~mm}$ for TAPSE; $p<0.001$ for all parameters except for LAD, $p=0.042$ ).

Regarding the severity of valvular heart diseases, the trainee's scan without tele-advice showed poor to moderate agreements, which were dramatically improved by tele-advice (Kappa coefficients; from 0.38 to 0.90 , from 0.21 to 0.85 , from 0.51 to 0.83 , and from 0.55 to 1.00 for aortic regurgitation, aortic stenosis, mitral regurgitation, and tricuspid regurgitation, respectively; Fig. 3).
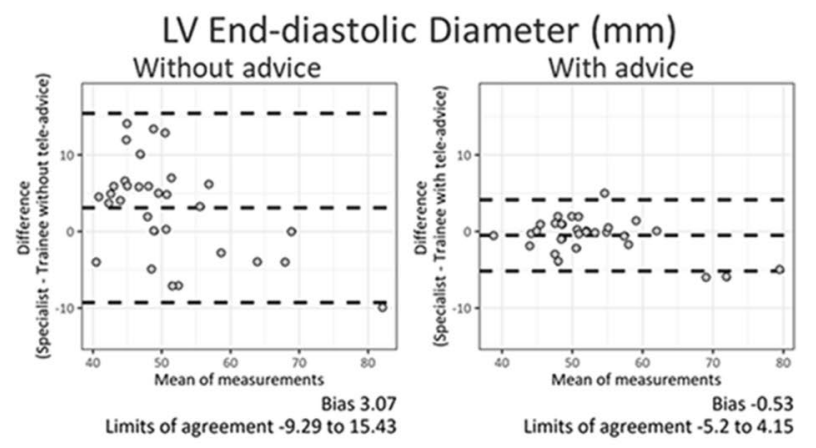

LV End-systolic Diameter ( $\mathrm{mm}$ )
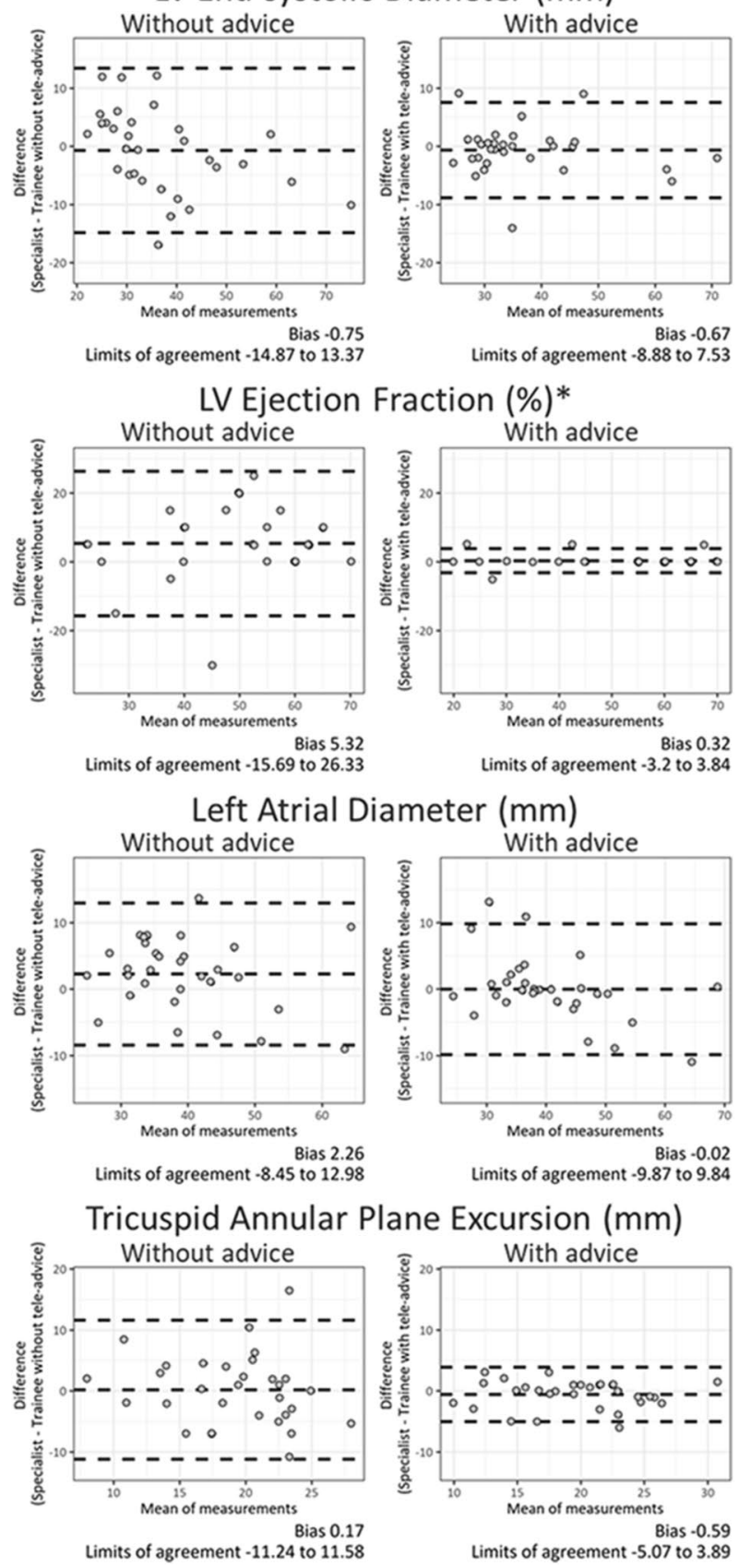


\section{Aortic regurgitation}
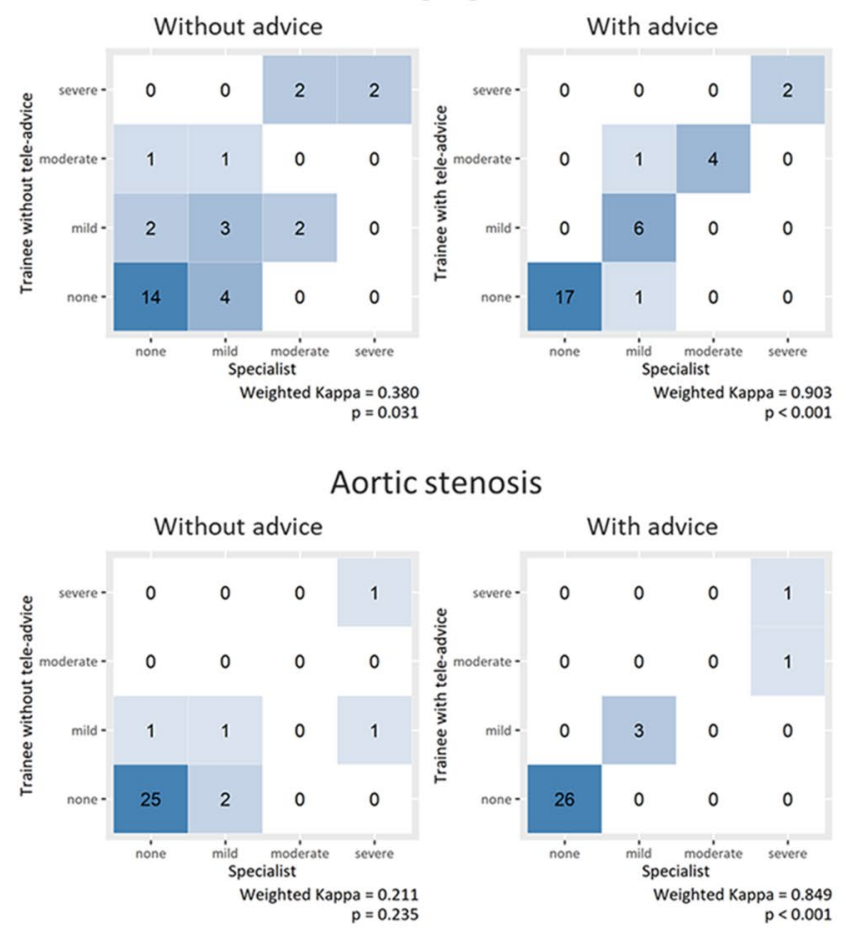

Fig. 3 Agreements in Valvular Heart Disease Severity. $x$ - and $y$-axes indicate measurements obtained by a specialist and a trainee, respectively. The left and right panels show the correlations in the absence

Importantly, the trainee's examination with tele-advice showed excellent accuracy for screening cardiac dysfunction, as demonstrated in Fig. 4. Under tele-advice, LV systolic function classification into preserved, midrange, and reduced LVEF (>50\%, 50-35\%, and $>35 \%$, respectively; we selected 35 as our cutoff value since several treatment indications are based on above and below 35) and significant valvular heart disease identification showed perfect agreement with the specialist's results. Identification of regional LV wall motion abnormality and abnormal right ventricular systolic function as abnormal TAPSE $(<17 \mathrm{~mm})$ were also improved by tele-advice (weighted Kappa coefficient, from 0.59 to 0.88 and from 0.50 to 0.77 , respectively). The presence of pericardial effusion was not correctly diagnosed by the trainees; the only patients who actually had pericardial effusion was overlooked, while another patient who did not have pericardial effusion was misdiagnosed as having pericardial effusion. With the tele-advice, the diagnosis of pericardial effusion also showed perfect agreement.
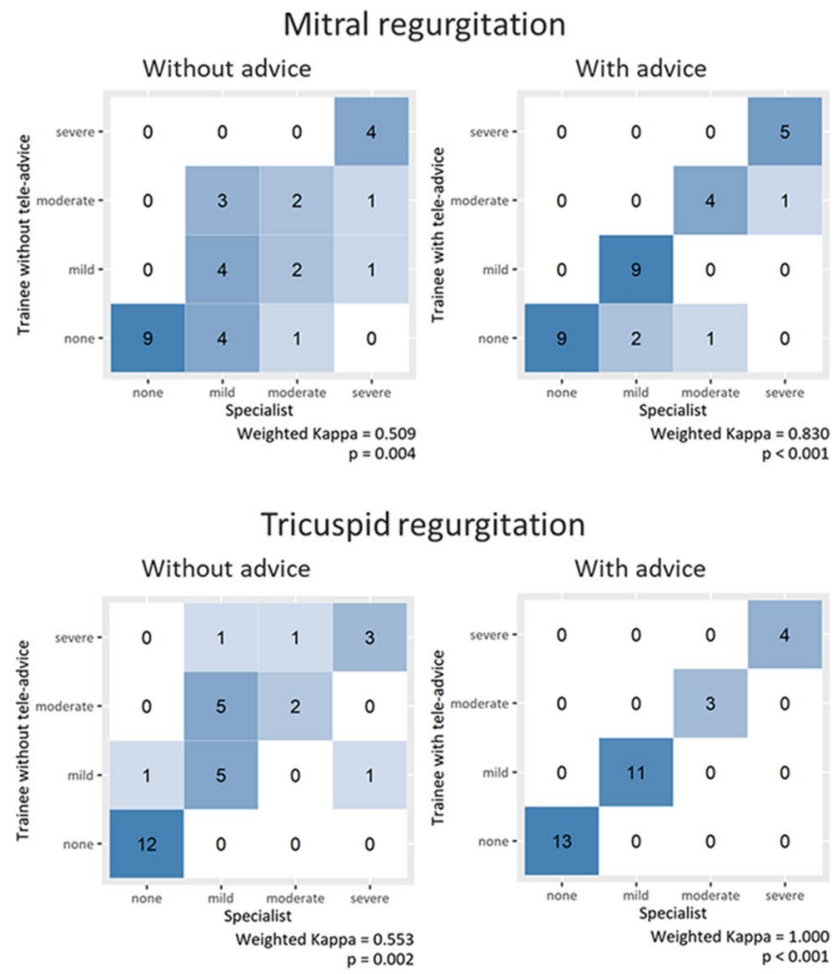

and presence of tele-ultrasound, respectively. Weighted Kappa coefficients improved in all parameters by tele-ultrasound

\section{Discussion}

To the best of our knowledge, this study is the first to demonstrate the efficacy of a tele-ultrasound system using a dedicated commercially available handheld ultrasound device. The overall study outline is summarized in Fig. 5. The results showed that tele-ultrasound enables the performance of specialist-level echocardiography even in settings where no echocardiography specialist is available.

Although ultrasound is one of the most established imaging modalities used in medical diagnosis, there is a substantial learning curve for performing and interpreting ultrasound examinations; moreover, the quality of ultrasound examinations varies significantly depending on the observer's experience, unlike computed tomography or magnetic resonance imaging, which provide very consistent and observer-independent images [18]. In addition, ultrasound examinations do not usually record all slices/ images of the target organ, unlike other imaging modalities, and unrecorded images can never be referred to remote doctors. Thus, there is a unique and significant demand for "real-time" telecommunication and/or supervision when performing ultrasound. Furthermore, the dynamic motion of the heart in movies carries crucial information in echocardiography [19]. Typical telecommunication tools, such as 

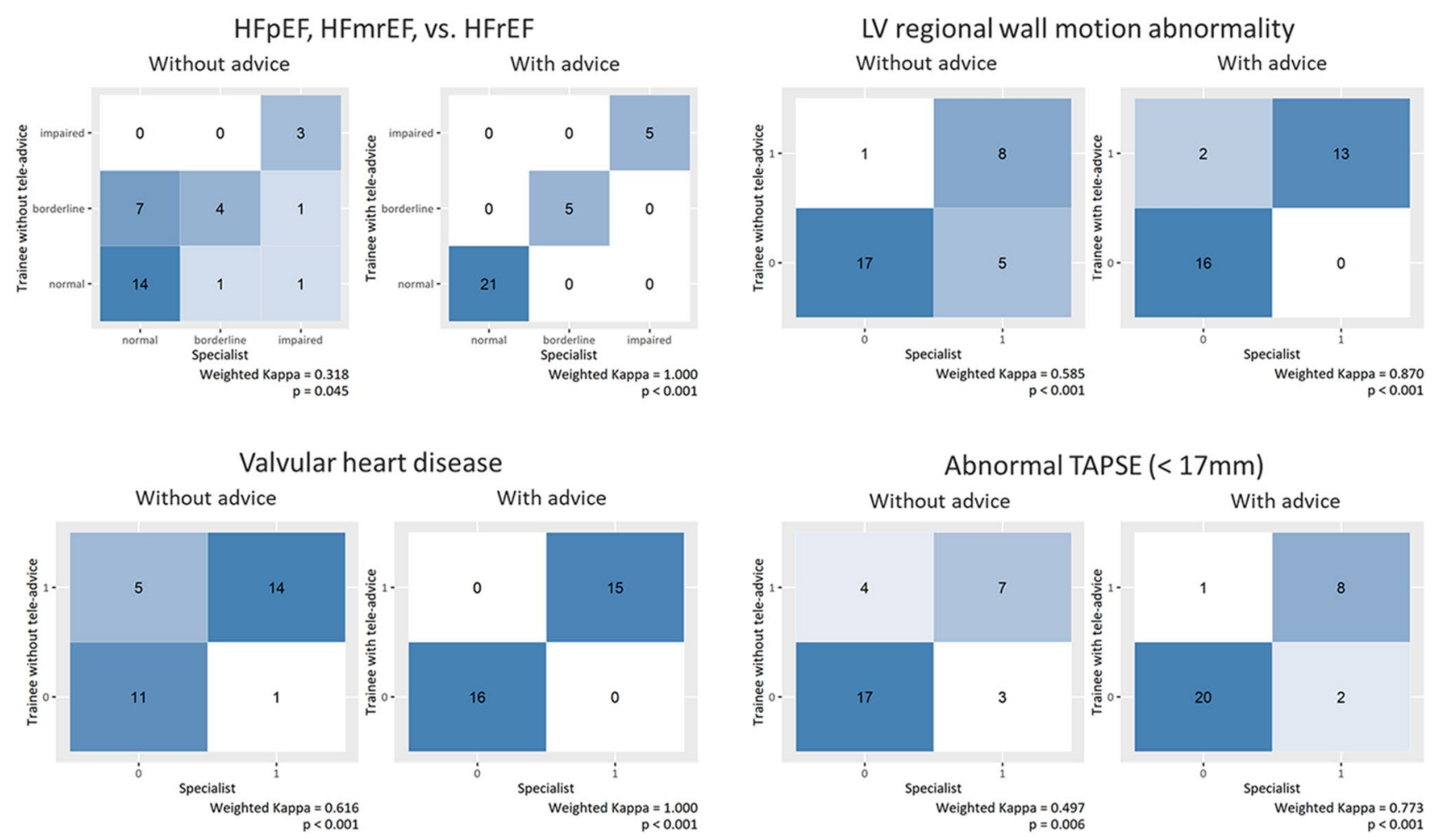

Fig. 4 Agreements in Simple Categorization. $x$ - and $y$-axes indicate measurements obtained by a specialist and a trainee, respectively. The left and right panels show the correlations in the absence and presence of tele-ultrasound, respectively. With tele-ultrasound, these simple screening measurements showed almost perfect agreements.

$H F r E F$ heart failure with reduced ejection fraction, $H F m r E F$ heart failure with mid-range ejection fraction, $H F p E F$ heart failure with preserved ejection fraction, TAPSE tricuspid annular plane systolic excursion

Fig. 5 Study Outline. The overall study outline is summarized. With tele-ultrasound, real-time tele-ultrasound using the handheld device enabled specialist-level echocardiography by non-specialists

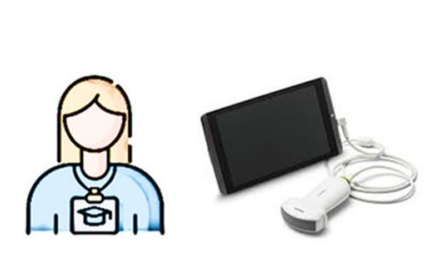

Trainee alone

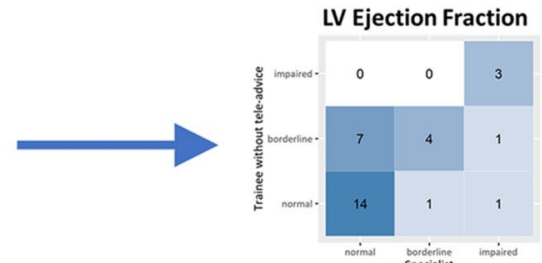

Moderate Accuracy

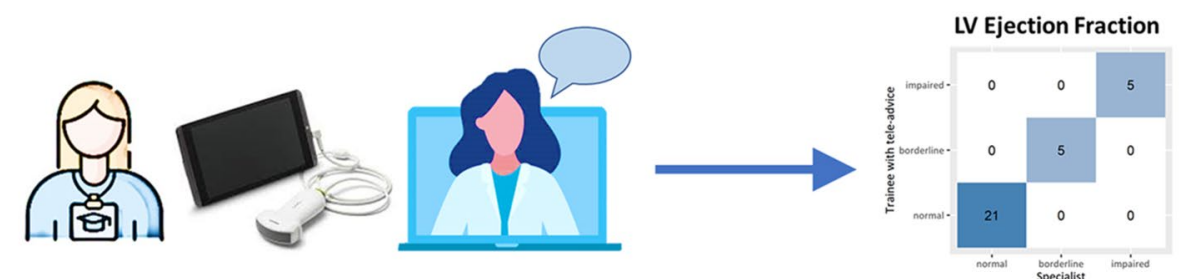

Trainee with tele-advice via the device

Perfect Accuracy

Zoom or FaceTime, do not usually provide a sufficient frame rate for accurate diagnosis in echocardiography.

In this study, we used a device that was developed specifically for tele-ultrasound, and thus was optimized for this purpose [8]. The ultrasound device enables telecommunication, and therefore the observer can focus on performing an examination since no other device is required. The observer and a remote doctor can simultaneously observe the images; 
therefore, the remote doctor can instruct the observer to acquire appropriate views and avoid overlooking important findings. The dedicated communication system attached to this device usually provides a sufficient frame rate for diagnosis, although it may depend on the internet connection. Furthermore, the remote doctor can control a pointer on the screen, which helps reduce miscommunication and greatly increases the accuracy of measurements by the observer.

There are many resource-limited medical teams and situations where such tele-ultrasound is useful as a specialist is not available. For example, coronavirus disease 2019 (COVID-19) is the most important topic in medicine today [20, 21]. This infectious disease frequently causes cardiovascular involvement, such as LV and right ventricular wall motion abnormalities, which are associated with a higher rate of in-hospital morbidity and mortality. In addition, the existence of baseline cardiovascular disease has been reported as a risk factor for adverse clinical outcomes [22-24]. However, many medical teams for COVID19 management do not involve an echocardiography specialist because medical care for patients with COVID-19 entails the risk of infection, and the number of medical staff who directly contact patients should be restricted [25, 26]. Importantly, our results showed that even trainees can provide specialist-level echocardiography examinations using this tele-ultrasound system. In particular, the almost perfect agreements in simple estimations of LV and right ventricular functions in our results highlight the efficacy of this system as a perfect screening tool, as these findings have been reported to be a marker of poor prognosis [22, 27]. Thus, tele-ultrasound is a valuable option for identifying cardiovascular complications in patients with COVID-19. Also, our results suggest that this system may be useful for education for trainees who have just started learning echocardiography, in consistency with the previous literature [28]. Further studies are warranted to establish the educational effect.

\section{Limitations}

Our study has several limitations. First, this was a singlecenter study with a relatively small number of patients. Second, the study was only conducted at Juntendo University Hospital, where a Wi-Fi connection was provided by the hospital. Although internet connectivity is becoming a common infrastructure in most developed countries, the system may not be feasible in places with an unstable internet connection. Next, this tele-ultrasound may be time-consuming and thus not be very useful for routine echocardiographic examinations. The primary use of this system may be limited in situations where a specialist is not available onsite or for educational purposes. Finally, our trainees were in their fellowship program and were not completely novice. The results may not be applied to a paramedical staff such as a nurse or a non-medical staff without medical knowledge.

\section{Conclusion}

Real-time tele-ultrasound using the handheld device was feasible and enabled specialist-level echocardiographic results by non-specialists. This approach might have a possibility of being useful in resource-limited medical situations and teams, such as rural areas and infectious disease teams.

Funding This work was partially supported by JSPS KAKENHI Grantin-Aid for Research Activity Start-up Grant Number 20K22504. The handheld ultrasound device (Lumify) and the tele-ultrasound system (Reacts) were provided by Philips. However, Philips was not involved in conducting this clinical study. Dr. Kagiyama is affiliated with a department funded by Philips Japan; Asahi KASEI Corporation; Inter Reha Co., Ltd; and Toho Holdings Co., Ltd based on collaborative research agreements.

\section{References}

1. Agarwal MA, Ziaeian B, Lavie CJ, Fonarow GC. Cardiovascular disease in hospitalized patients with a diagnosis of coronavirus from the pre-COVID-19 era in united states: national analysis from 2016-2017. Mayo Clin Proc. 2020;95:2674-83.

2. Mensah GA, Brown DW. An overview of cardiovascular disease burden in the United States. Health Aff. 2007;26:38-48.

3. Wiegers SE, Ryan T, Arrighi JA, Brown SM, Canaday B, Damp JB, et al. 2019 ACC/AHA/ASE Advanced training statement on echocardiography (revision of the 2003 ACC/AHA clinical competence statement on echocardiography): a report of the ACC Competency Management Committee. J Am Coll Cardiol. 2019;74:377-402.

4. Bashshur R, Shannon G, Krupinski E, Grigsby J. The taxonomy of telemedicine. Telemed J e-Health. 2011;17:484-94.

5. Meyer BC, Raman R, Hemmen T, Obler R, Zivin JA, Rao R, et al. Efficacy of site-independent telemedicine in the STRokE DOC trial: a randomised, blinded, prospective study. Lancet Neurol. 2008;7:787-95.

6. Moskowitz A, Chan YF, Bruns J, Levine SR. Emergency physician and stroke specialist beliefs and expectations regarding telestroke. Stroke. 2010;41:805-9.

7. Chamsi-Pasha Mohammed A, Sengupta Partho P, Zoghbi WA. Handheld echocardiography. Circulation. 2017;136:2178-88.

8. Philips. Philips Lumify powered by Reacts. 2020

9. Britton N, Miller MA, Safadi S, Siegel A, Levine AR, McCurdy MT. Tele-ultrasound in resource-limited settings: a systematic review. Front Public Health. 2019;7:244.

10. Levine AR, Verceles AC, McCurdy MT. A simple but often ignored formula to improve critical care: telemedicine + ultrasound $=$ tele-ultrasound. Crit Care Med. 2020;48:e540. 
11. Kusunose K, Shibayama K, Iwano H, Izumo M, Kagiyama N, Kurosawa K, et al. Reduced variability of visual left ventricular ejection fraction assessment with reference images: The Japanese Association of Young Echocardiography Fellows multicenter study. J Cardiol. 2018;72:74-80.

12. Thavendiranathan P, Popović ZB, Flamm SD, Dahiya A, Grimm RA, Marwick TH. Improved interobserver variability and accuracy of echocardiographic visual left ventricular ejection fraction assessment through a self-directed learning program using cardiac magnetic resonance images. J Am Soc Echocardiogr. 2013;26:1267-73.

13. Izumi C, Eishi K, Ashihara K, Arita T, Otsuji Y, Kunihara T, et al. JCS/JSCS/JATS/JSVS 2020 Guidelines on the management of valvular heart disease. Circ J. 2020;84:2037-119.

14. Lang RM, Badano LP, Mor-Avi V, Afilalo J, Armstrong A, Ernande L, et al. Recommendations for cardiac chamber quantification by echocardiography in adults: an update from the American Society of Echocardiography and the European Association of Cardiovascular Imaging. J Am Soc Echocardiogr. 2015;28:1-39 e14.

15. Nishimura RA, Otto CM, Bonow RO, Carabello BA, Erwin JP 3rd, Fleisher LA, et al. 2017 AHA/ACC focused update of the 2014 AHA/ACC guideline for the management of patients with valvular heart disease: a report of the american college of cardiology/american heart association task force on clinical practice guidelines. Circulation. 2017;135:e1159-95.

16. Gamer M. Package 'irr'. 2012

17. Lehnert B. Package 'BlandAltmanLeh'. 2015

18. Morbach C, Gelbrich G, Breunig M, Tiffe T, Wagner M, Heuschmann PU, et al. Impact of acquisition and interpretation on total inter-observer variability in echocardiography: results from the quality assurance program of the STAAB cohort study. Int J Cardiovasc Imaging. 2018;34:1057-65.

19. Sutherland GR, Kukulski T, Kvitting JE, D’Hooge J, Arnold M, Brandt E, et al. Quantitation of left-ventricular asynergy by cardiac ultrasound. Am J Cardiol. 2000;86:4G-9G.

20. Hollander JE, Carr BG. Virtually perfect? Telemedicine for Covid-19. N Engl J Med. 2020;382:1679-81.
21. Keesara S, Jonas A, Schulman K. Covid-19 and health care's digital revolution. N Engl J Med. 2020;382:e82.

22. Dweck MR, Bularga A, Hahn RT, Bing R, Lee KK, Chapman AR, et al. Global evaluation of echocardiography in patients with COVID-19. Eur Heart J Cardiovasc Imaging. 2020;21:949-58.

23. Mehra MR, Desai SS, Kuy S, Henry TD, Patel AN. Cardiovascular disease, drug therapy, and mortality in covid-19. N Engl J Med. 2020;382:e102.

24. Sud K, Vogel B, Bohra C, Garg V, Talebi S, Lerakis S, et al. Echocardiographic findings in patients with COVID-19 with significant myocardial injury. J Am Soc Echocardiogr. 2020;33:1054-5.

25. Nicoara A, Maldonado Y, Kort S, Swaminathan M, Mackensen GB. Specific considerations for the protection of patients and echocardiography service providers when performing perioperative or periprocedural transesophageal echocardiography during the 2019 novel coronavirus outbreak: council on perioperative echocardiography supplement to the statement of the american society of echocardiography endorsed by the society of cardiovascular anesthesiologists. J Am Soc Echocardiogr. 2020;33:666-9.

26. Seo Y, Daimon M, Yamada H, Kagiyama N, Ohta M, Izumi C, et al. Review of the efforts of the Japanese Society of Echocardiography for coronavirus disease 2019 (COVID-19) during the initial outbreak in Japan. J Echocardiogr. 2020;18:226-33.

27. Giustino G, Croft LB, Stefanini GG, Bragato R, Silbiger JJ, Vicenzi M, et al. Characterization of myocardial injury in patients with COVID-19. J Am Coll Cardiol. 2020;76:2043-55.

28. Yamada S. Possibilities of online communication tools for dissemination and education of point-of-care ultrasound. Choonpa Igaku. 2018;46

Publisher's Note Springer Nature remains neutral with regard to jurisdictional claims in published maps and institutional affiliations. 